\title{
Diseño de un prototipo de trituradora para mejorar el rendimiento de trituración de botellas tipo PET
}

\section{Desing of a crusher prototype to improve the crushing performance of type PET bottles}

\author{
'Gamarra Moreno, Arturo Huber; Taipe Castro, José Antonio; Arellano Vílchez, Mario Alfonso \\ 2Lozano Paulino, Yovany Damisela; ${ }^{3}$ Samaniego Valdéz, Percy Roger; ${ }^{3}$ Serpa Enríquez, Christian Andrés \\ Facultad de Ingeniería Mecánica, Universidad Nacional del Centro del Perú \\ Email: ahgamarra@uncp.edu.pe
}

\section{Resumen}

El estudio realizado corresponde al tipo de investigación tecnológica, ya que mediante la aplicación de las metodologías de diseño mecánico, se buscó mejorar el rendimiento de una trituradora de botellas tipo PET; el nivel de investigación es experimental, debido a que considerando el tipo de geometría de la cuchilla de corte, se buscó determinar aquel que proporciona mayor rendimiento de trituración. El objetivo de esta investigación fue diseñar un prototipo de trituradora cuya geometría de cuchilla de corte permita mejorar el rendimiento de trituración de botellas tipo PET. Considerando la metodología de diseño VDI 2221, se elaboró la lista de exigencias para el diseño de un prototipo de una máquina trituradora de plásticos PET y la matriz morfológica correspondiente y se obtuvo resultados cualitativos que indican que el rendimiento de trituración está en función de la geometría de la cuchilla de corte de la trituradora de botellas tipo PET. Así mismo, se utilizó el cálculo de diseño mecánico para seleccionar los componentes del prototipo de trituradora, el resultado cuantitativo de este estudio realizado requirió de las mediciones de rendimiento de trituración según el tipo de geometría las cuchillas de corte, luego mediante el análisis de la varianza (ANOVA) de un factor, se determinó que para un nivel de confianza del $95 \%$ el tipo de geometría C (cuchilla con 3 uñas) proporciona mayor rendimiento de trituración con un valor promedio de $21.72 \mathrm{~kg} / \mathrm{h}$.

Palabras claves: Diseño, trituradora de plásticos PET, rendimiento de trituración.

\begin{abstract}
The investigation corresponds to technological research type, because the application of mechanical design methodologies sought improving the performance of grinding crusher of bottles PET type; the research level is experimental, because on the basis of the of the cutting blade geometry type it was sought to determine the one with greater grinding performance. The objective of this study was to design a prototype whose cutting blade geometry improves the crushing performance of bottles type PET. Considering the Design Methodology VDI 2221 the list of requirements for the design of a prototype of a crushing machine plastic PET and the corresponding morphological matrix were developed and the obtained qualitative results indicate that the crushing performance is in function of the geometry of the cutting blade of bottle type PET crusher. Additionally, mechanical design calculation helped to select the components of the prototype crusher, the quantitative result of this study required crushing performance measurements according to the geometry type of cutting blades, then by analysis of variance (ANOVA) of one factor it was determined that for a confidence level of $95 \%$ the geometry $\mathrm{C}$ type (blade with three nails) provides higher trituration yield with an average value of $21.72 \mathrm{~kg} / \mathrm{h}$.
\end{abstract}

Keywords: Design, PET plastic crusher, crushing performance. 


\section{Introducción}

Uno de los mayores problemas del mundo actual es que la basura que se origina es de tal volumen y capacidad que no hay tiempo suficiente para tratarla; sin embargo, se encuentran muchas empresas que están haciendo la diferencia al emplear en su producción un material que se desecha todos los días, botellas PET (Tereftalato de polietileno) al igual que, también, latas de aluminio (gaseosas, refrescos y cervezas). En el Perú, se genera aproximadamente 18 mil 870 toneladas de basura, a dicha cifra, toda la región de Junín contribuye con 479 toneladas. Además, un estudio realizado por el Ministerio del Ambiente revela que Huancayo está entre las 10 ciudades del país que generan más residuos sólidos.

El PET es un poliéster, un polímetro termoplástico. Es el sobresaliente y más resistente plástico para la producción de botellas para agua, jugos, refresco, aceites y otros. Son $100 \%$ reciclables y las botellas PET pueden separarse de distintas maneras, las máquinas que se encargan de triturarlos o molerlos utilizan cuatro métodos: compresión, impacto, frotamiento por cizalla y corte.

Teniendo en cuenta lo expuesto anteriormente y buscando el aprovechamiento del PET mediante el reciclado a través del proceso de trituración en esta investigación se propuso el diseño de un prototipo de trituradora con herramienta de corte, debido a que es necesario contar con máquinas con buen rendimiento que permitan el reciclaje de desechos (PET); contribuyendo así, a superar el problema ambiental que causan la presencia de los mismos.

Por lo tanto, el objetivo general de esta investigación es diseñar un prototipo de trituradora cuya geometría de cuchilla de corte permita mejorar el rendimiento de trituración de botellas tipo PET; para ello, es necesario considerar las siguientes etapas: diseñar y construir un prototipo de máquina para trituración de botellas tipo PET, medir el rendimiento de trituración considerando 4 tipos de geometría de cuchillas de corte: 01$)$ Tipo A (cuchilla de corte de 9 uñas paralelas al eje de rotación); Tipo B (03 cuchillas de corte paralelas al eje de rotación); 03) Tipo C (cuchilla de corte de 3 uñas de discos independientes) y Tipo D (cuchilla de corte de 6 uñas de discos independientes); luego determinar cuál es la geometría de la cuchilla de corte que mejor rendimiento de trituración de botellas tipo PET ofrece. La idea es que se consiga el elemento recolectado, luego pase por un proceso de trituración por cuchillas para así poder reducir el volumen de almacenamiento y luego distribuir de una mejor y adecuada manera a las empresas encargadas de continuar con el proceso de reciclaje, teniendo en cuenta la energía requerida para la trituración.
También para la elaboración del prototipo se considera muchas características, tanto como del elemento a triturar, como también de la maquina prototipo, en función a la manera de cómo alimentar, como triturar, los distintos mecanismos que intervienen en su funcionamiento y como también la factibilidad de fabricación. Para el diseño de la trituradora se hace uso de la norma VDI 2221 la cual permite obtener varias alternativas de solución y determinar el diseño óptimo de la trituradora en función del tipo de $\mathrm{cu}$ chilla de corte utilizado; también se utiliza el cálculo de diseño mecánico para seleccionar los componentes del prototipo de trituradora, se procede con las mediciones de rendimiento de trituración según el tipo de geometría las cuchillas de corte, luego mediante el análisis de la varianza (ANOVA) de un factor se determina el tipo de geometría que proporciona mayor rendimiento en $\mathrm{kg} / \mathrm{h}$.

\section{Metodología}

\section{Materiales y métodos}

Los materiales requeridos para el desarrollo de la investigación, se centran fundamentalmente en documentos, archivos y elementos que contribuyeron con el diseño y construcción de un prototipo de trituradora de botellas tipo PET.

\section{Tipo de investigación}

El estudio realizado corresponde al tipo de investigación tecnológica, porque mediante la aplicación de las metodologías de diseño mecánico se buscó mejorar el rendimiento de la producción de una trituradora de botellas tipo PET; el nivel de investigación es experimental, debido a que considerando el tipo de geometría de la cuchilla de corte se buscó determinar aquel que proporciona mayor rendimiento de trituración.

La metodología que se llevó a cabo para la elaboración del presente trabajo de investigación tuvo en cuenta los procesos de diseño en ingeniería mecánica que en este caso incluye el diseño de elementos de máquina y la norma VDI 2221.

\section{Unidad de estudio}

Prototipo de trituradora de botellas tipo PET.

\section{Técnicas de procesamiento de datos}

Básicamente se recopiló información de rendimiento de trituración; es decir, para cada una de las geometrías “A”, "B”, “C” y “D”, consideradas en este estudio, se realizó 10 mediciones del rendimiento $(\mathrm{kg} / \mathrm{h})$ de trituración, tal como se indica en el cuadro 1, para todos los casos se controló la velocidad de $86 \mathrm{rpm}$. 
Cuadro 1: Rendimiento según tipo de geometría

\begin{tabular}{|c|c|c|}
\hline $\mathrm{N}^{\circ}$. Medición & $\begin{array}{l}\text { Tipo de geometria } \\
\text { de cuchilla de } \\
\text { corte }\end{array}$ & $\begin{array}{l}\text { Rendimiento } \\
(\mathbf{k g} / \mathrm{h})\end{array}$ \\
\hline 1 & $A$ & 10,5 \\
\hline 2 & $A$ & 12,6 \\
\hline 3 & A & 10,8 \\
\hline 4 & $A$ & 13,2 \\
\hline 5 & $A$ & 12.0 \\
\hline 6 & A & 14,1 \\
\hline 7 & A & 10,5 \\
\hline 8 & A & 12,6 \\
\hline 9 & A & 14,4 \\
\hline 10 & A & 11,4 \\
\hline 11 & B & 21.0 \\
\hline 12 & B & 17,4 \\
\hline 13 & B & 18,3 \\
\hline 14 & $\mathrm{~B}$ & 15,6 \\
\hline 15 & B & 18.0 \\
\hline 16 & B & 20,4 \\
\hline 17 & B & 17,1 \\
\hline 18 & B & 18,3 \\
\hline 19 & $B$ & 19,5 \\
\hline 20 & $B$ & 15,6 \\
\hline 21 & $\mathrm{C}$ & 22,5 \\
\hline 22 & C & 20,4 \\
\hline 23 & $\mathrm{C}$ & 24.0 \\
\hline 24 & C & 21,6 \\
\hline 25 & $\mathrm{C}$ & 20,7 \\
\hline 26 & C & 17,7 \\
\hline 27 & $\mathrm{C}$ & 21.0 \\
\hline 28 & $\mathrm{C}$ & 22,8 \\
\hline 29 & C & 24,6 \\
\hline 30 & $\mathrm{C}$ & 21,9 \\
\hline 31 & D & 15,6 \\
\hline 32 & $\mathrm{D}$ & 14,1 \\
\hline 33 & $\mathrm{D}$ & 15,6 \\
\hline 34 & $\mathrm{D}$ & 11,4 \\
\hline 35 & $\mathrm{D}$ & 14,7 \\
\hline 36 & D & 17,4 \\
\hline 37 & $\mathrm{D}$ & 12,6 \\
\hline 38 & $\mathrm{D}$ & 11,7 \\
\hline 39 & $\mathrm{D}$ & 14,7 \\
\hline 40 & D & 16,2 \\
\hline
\end{tabular}

Fuente: Elaboración propia

\section{Resultados}

Matriz morfológica

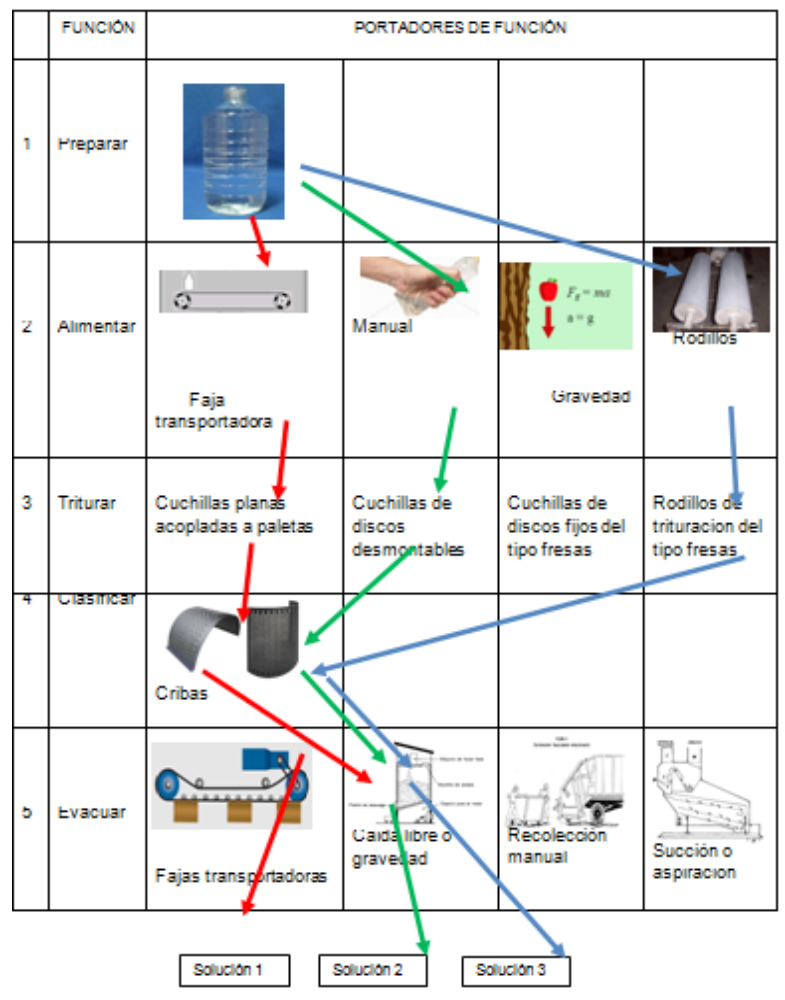

Solución $\mathrm{N}^{\mathbf{0}} 1$ : Trituradora de paletas y cuchillas planas

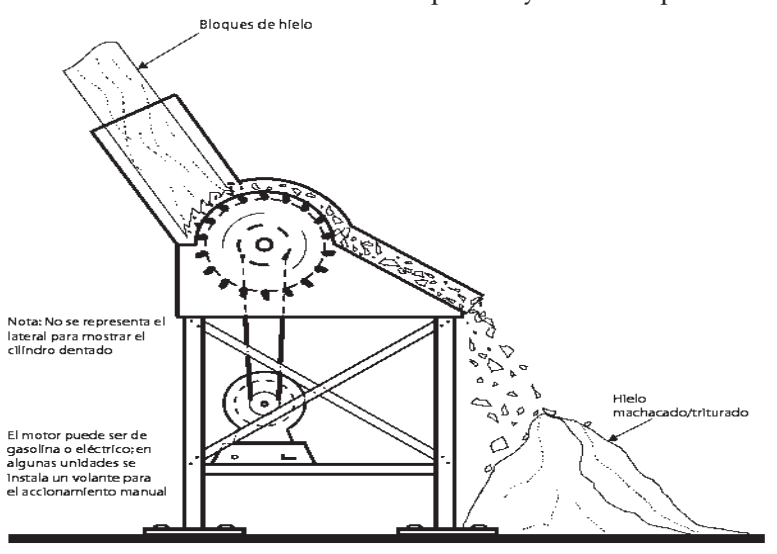

Solución $\mathbf{N}^{\circ}$ 2: Trituradora de discos desmontables 


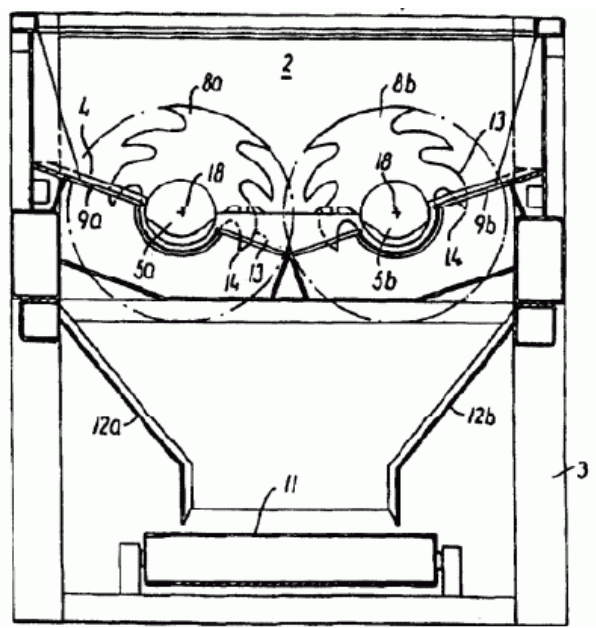

Solución $\mathrm{N}^{\mathbf{0}}$ 3: Trituradora de rodillos del tipo fresas

\section{Criterios de evaluación}

1: malo 2: regular 3: bueno

\begin{tabular}{lccc}
\hline & \multicolumn{3}{c}{ Soluciones } \\
\cline { 2 - 4 } Criterios técnicos y económicos & 1 & 2 & 3 \\
\hline \hline Estabilidad & 3 & 3 & 3 \\
\hline \hline Número de operaciones & 2 & 3 & 2 \\
\hline \hline Facilidad de manejo & 2 & 3 & 2 \\
\hline \hline Costo de tecnología & 1 & 3 & 2 \\
\hline \hline Seguridad & 3 & 3 & 3 \\
\hline \hline Facilidad de mantenimiento y montaje & 1 & 3 & 2 \\
\hline \hline Rapidez de trabajo & 3 & 3 & 3 \\
\hline \hline Suma total & 15 & 21 & 17 \\
\hline \hline
\end{tabular}

Con referencia al criterio de evaluación, se tiene que la solución que obtuvo mejor puntaje es la solución $\mathrm{N}^{\mathrm{o}} 2$, la cual será de referencia para desarrollar, diseñar y fabricar nuestro prototipo.

Tal como se muestra en la matriz morfológica, se realizó el diseño de la trituradora según la norma VDI 2221, lo cual permitió obtener varias alternativas de solución, siendo la óptima el diseño que considera el tipo de geometría de la herramienta de corte, el tipo "C" por presentar ventajas de agarre del plástico, corte del plástico, capacidad de producción y un menor tiempo de triturado con respecto a los otros tipos de geometría “A”, “B” y "D” consideradas en este estudio.

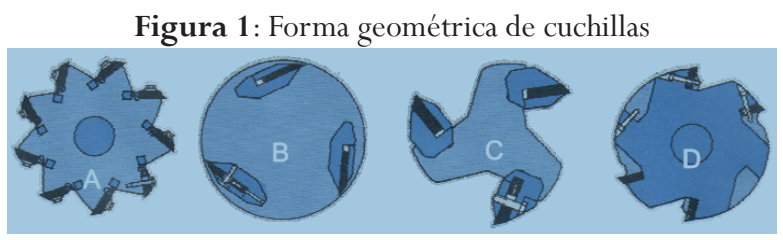

Según los criterios y cálculos de diseño, se determinó la potencia del motoreductor igual a $1 \mathrm{hp}$, se eligió los rodamientos rígidos de bolas de la serie 62, cuyo número es el 6204-2Z (SKF), por ser más económico y ser más comercial. Por tanto, el diámetro del eje del órgano de trabajo fue de $20 \mathrm{~mm}$.

Luego de seleccionar la mejor solución a partir de la matriz morfológica mostrada anteriormente, en el cuadro 2, se puede ver el resumen de las pruebas con las geometrías de cuchillas probadas en dicha solución, teniendo en cuenta la metodología de diseño según la norma VDI 2221.

Cuadro 2: Evaluación del performance de las formas geométricas de las cuchillas

\begin{tabular}{lcccc}
\hline \multirow{2}{*}{ Parámetros } & \multicolumn{4}{c}{ Cuchillas } \\
\cline { 2 - 5 } & A & B & C & D \\
\hline \hline Agarre del plástico & 2 & 3 & 4 & 2 \\
\hline \hline Corte del plástico & 4 & 4 & 4 & 4 \\
\hline \hline Capacidad de producción & 2 & 3 & 4 & 2 \\
\hline \hline Tiempo de triturado & 2 & 3 & 4 & 2 \\
\hline \hline & 10 & 13 & 16 & 10 \\
\hline \hline
\end{tabular}

Fuente: Elaboración propia

\section{Hipótesis estadística}

$\mathrm{H}_{0}$ : Hipótesis nula

$\mathrm{H}_{1}$ : Hipótesis alterna

Cuadro 3: Descriptivos del rendimiento de cuchillas de corte

\begin{tabular}{cccccc}
\hline \hline & & & & \multicolumn{2}{c}{$\begin{array}{c}\text { 95\% del intervalo } \\
\text { de confianza para } \\
\text { la media }\end{array}$} \\
\cline { 5 - 6 } & N & Media & $\begin{array}{c}\text { Error } \\
\text { estándar }\end{array}$ & $\begin{array}{c}\text { Límite } \\
\text { inferior }\end{array}$ & $\begin{array}{c}\text { Límite } \\
\text { superior }\end{array}$ \\
\hline \hline A & 10 & 12,210 &, 4496 & 11,193 & 13,227 \\
B & 10 & 18,120 &, 5748 & 16,820 & 19,420 \\
C & 10 & 21,720 &, 6216 & 20,314 & 23,126 \\
D & 10 & 14,400 &, 6229 & 12,991 & 15,809 \\
Total & 40 & 16,613 &, 6425 & 15,313 & 17,912 \\
\hline \hline
\end{tabular}

Fuente: Resultados en SPSS 


\section{Análisis de la varianza}

Cuadro 4: Análisis de la varianza de rendimientos de cuchillas de corte

ANOVA

Rendimiento $(\mathrm{kg} / \mathrm{h})$

\begin{tabular}{llllll}
\hline \hline & $\begin{array}{c}\text { Suma } \\
\text { de cua- } \\
\text { drados }\end{array}$ & gl & $\begin{array}{c}\text { Media } \\
\text { cuadrá- } \\
\text { tica }\end{array}$ & F & Sig. \\
\hline $\begin{array}{l}\text { Entre } \\
\text { grupos }\end{array}$ & 526,363 & 3 & 175,454 & 53,701 &, 000 \\
\hline \hline $\begin{array}{l}\text { Dentro de } \\
\text { grupos }\end{array}$ & 117,621 & 36 & 3,267 & \\
\hline \hline Total & 643,984 & 39 & & \\
\hline
\end{tabular}

Fuente: Resultados en SPSS

\section{Prueba post hoc}

Cuadro 5: Determinación de geometría de cuchillas de corte que proporciona mayor rendimiento de trituración

\section{Rendimiento (kg/h)}

HSD Tukey

\begin{tabular}{|c|c|c|c|c|c|}
\hline \multirow{2}{*}{$\begin{array}{l}\text { Tipo de } \\
\text { cuchilla }\end{array}$} & \multirow{2}{*}{$\mathbf{N}$} & \multicolumn{4}{|c|}{ Subconjunto para alfa $=0.05$} \\
\hline & & 1 & 2 & 3 & 4 \\
\hline A & 10 & 12,210 & & & \\
\hline $\mathrm{D}$ & 10 & & 14,400 & & \\
\hline B & 10 & & & 18,120 & \\
\hline C & 10 & & & & 21,720 \\
\hline Sig. & & 1,000 & 1,000 & 1,000 & 1,000 \\
\hline
\end{tabular}

Se visualizan las medias para los grupos en los subconjuntos homogéneos.

a. Utiliza el tamaño de la muestra de la media armónica $=10,000$.

\section{Gráficos}

Figura 2: Rendimiento según tipo de geometría de cuchillas de corte

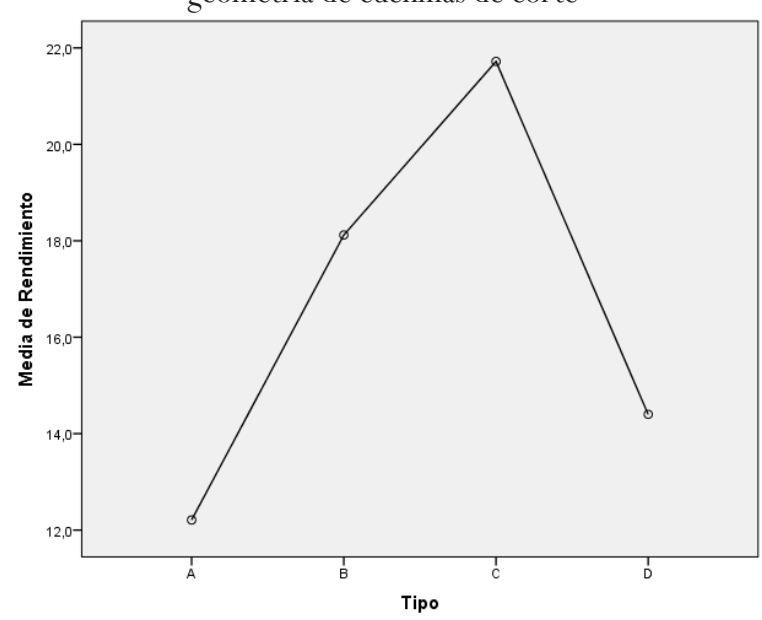

Del análisis de la varianza ANOVA realizada, se obtuvo que para un nivel de significancia del $5 \%$ existe diferencia significativa entre los rendimientos de trituración (en $\mathrm{kg} / \mathrm{h}$ ) según la geometría de las cuchillas de corte considerados en esta investigación.

A partir de la prueba post hoc realizada, se concluye que el tipo de geometría "C" de la cuchilla de corte proporciona un mayor rendimiento en comparación con las otros tres tipos de geometría “A”, "B”" y "D" respectivamente considerados en esta investigación.

\section{Discusión}

Según los resultados del cuadro 2, trabajando a la velocidad de operación de $86 \mathrm{rpm}$, la cuchilla de 3 uñas (tipo C) es la que rinde más porque los $\mathrm{kg} / \mathrm{h}$ de trituración es mayor con respecto a las cuchillas de tipos de geometría A, B y D respectivamente, lo cual es corroborada con el análisis de la varianza ANOVA indicada en el cuadro 4 que muestra que para un nivel de significancia del 5\% existe diferencia significativa entre los rendimientos de trituración según la geometría de las cuchillas de corte, además, en la el cuadro 5 y la Figura 2 se puede observar que el tipo de geometría $\mathrm{C}$ de la cuchilla de corte (cuchilla de 3 uñas) proporciona un mayor rendimiento en comparación con las otros tres tipos de geometría.

$\mathrm{Al}$ igual que lo considerado en la investigación titulada "Diseño y construcción de un equipo triturador de botellas plásticas tipo PET" desarrollado por Freire y Gonzáles. El sistema de alimentación en este caso es manual y se eligió la herramienta de corte basado en cuchillas, pues se coincide en que las máquinas con cuchillas cortadoras rotatorias son las más comunes en lo referido a máquinas para moler plástico.

Medina, Saldaña, Hernández y Becerra (2011, p. 516) en su trabajo "Diseño de una trituradora para plástico Polietileno de Tereftalato (PET)" también señalan que la molienda del Polietileno de Tereftalato (PET), se realiza dentro de un molino de cuchillas giratorias, las cuales tienen como función triturar las botellas hasta convertirlas en hojuelas con dimensiones específicas; de igual modo, en esta investigación se consideró como herramienta de corte las cuchillas giratorias.

La obtención de los resultados del rendimiento de trituración de esta investigación considerada en el ANOVA tuvo en cuenta sólo un factor (Tipo de geometría de corte) en este caso que, por lo que futuros estudios podrían considerar otros factores relacionados con la herramienta de corte u otros componentes de la trituradora que seguramente tienen influencia en el rendimiento de trituración, ello contribuirá a una análisis más exhaustivo en la obtención del rendimiento de trituración. 


\section{Referencias bibliográficas}

Camenza, Y. (2011). UNAD. Retrieved Junio 8, 2016, from UNAD: http: / / datateca.unad.edu.co/contenidos/358012/ContLin/leccin_1_los_residuos_ slidos.html

Freire, L., \& Gonzáles, C. (2013). Diseño y construcción de un equipo triturador de botellas plásticas tipo PET. Río Bamba Ecuador.

García, G. (1984). Un proceso general de diseño en Ingeniería Mecánica. Dialnet , 35-43.

Hori, J. (2002). Diseño de elementos de máquina. Lima Perú.

Interempresas. (2014, Mayo 20). Interempresas net. Retrieved 01 2017, 18, from Interempresas net: http: / / www.interempresas.net/Plastico/Articulos/123244-Molinos-no-solo-granular-o-triturar. html

Jänsch, J., \& Birkhofer, H. (2006, Mayo 15 - 18). The development of the guideline - The change of direction. Theory and research methods in design - International design Conference - Design 2006, 45-52.

Medina, J., Saldaña, C., Hernández, V., \& Becerra, S. (2011). Diseño de una trituradora para plástico Polietileno de Tereftalato (PET). Hacia la sustentabilidad: Los residuos sólidos como fuente de energía y materia prima, 514-518.

PUCP. (2016, Noviembre 14). Reciclando plástico PET en el Perú. Retrieved Enero 03, 2017, from Clima de cambios PUCP: http://www.pucp.edu.pe/climadecambios $/$ index.php?tmpl=articulo\&id $=2086$

Sandoval, D., \& Ushiña, V. (2011). Diseño y construcción de un prototipo de máquina recicladora de botellas plásticas PET de 400 a $500 \mathrm{ml}$ y latas de aluminio de 330 a $350 \mathrm{ml}$. Proyecto previo a la obtención del título de Ingeniero Mecánico, Escuela Politécnica del Ejército, Carrera de Ingeniería Mecánica, Sangolquí, Ecuador.

Serrano, M., \& Urdaneta, L. (2009). Diseño de una máquina destructoras de gomas (elastómeros) para frenos en desechos, de la empresa AKRON gomas de Venezuela S.A. Universidad Nacional Experimental del Táchira, Táchira - Venezuela. 